\title{
2. EXECUTIVE SUMMARY
}

This is the ninth report on mothers and babies in NSW to combine the annual reports of the NSW Midwives Data Collection (MDC), the Neonatal Intensive Care Units' Data Collection (NICUS), and the NSW Birth Defects Register (BDR).

In view of the substantial increase in births observed in 2005, this report includes a supplementary chapter (Chapter 13) on demographic trends in births in NSW.

Information on causes of maternal deaths in NSW was obtained through the work of the NSW Maternal and Perinatal Committee. From 1 January 2000, confidential reviews of perinatal deaths among babies of at least 22 weeks gestation or 500 grams birthweight are also carried out by the Committee. Chapter 12 describes the results of the review for deaths occurring in 2005.

\section{Trends in NSW}

Between 2001 and 2004, the numbers of births in NSW remained stable at about 86,000 per annum. In 2005, the number of births increased to 90,610 , a rise of 5.8 per cent compared to 2004 .

The increase in births occurred mainly in the Sydney area, with both the Sydney South West and Sydney West Areas reporting over 1,000 more births in 2005 than 2004.

The largest increase in births occurred among mothers aged 35-39 years. While the number of births to teenage mothers was slightly higher in 2005 compared to 2004, the rate of increase was less than other maternal age groups, and the overall percentage of births to teenage mothers followed the trend of previous years, falling slightly from 4.0 per cent of all births in 2004 to 3.9 per cent in 2005 .

Births to Aboriginal and Torres Strait Islander mothers also increased in 2005, with most of the increase occurring among mothers aged 20-29 years. Births to teenage mothers followed the same pattern as for non-Aboriginal mothers, with the number of births increasing slightly and the overall proportion of births to teenage mothers following the downward trend of previous years.

The increase in births in 2005 occurred fairly evenly among mothers who were giving birth for the first time and mothers who had given birth previously. There was no substantial change in the numbers of previous pregnancies among mothers who gave birth in 2005 compared to previous years.

Following the pattern of previous years, about 28 per cent mothers were born overseas in 2005, most commonly in the United Kingdom (2.7 per cent), New Zealand (2.5 per cent), China (2.0 per cent) and Vietnam (1.9 per cent).

Between 2001 and 2005, the proportion of mothers planning to give birth in a birth centre remained stable at 3-4 per cent, while the reported number of mothers planning a home birth fell from 182 to 152 over the 5 year period.

The rate of normal vaginal birth fell from 65.4 per cent in 2001 to 61.2 per cent in 2005 . Over the 5 years, the caesarean section rate increased from 23.6 to 28.1 per cent and the rate of instrumental delivery remained steady at 10 to 11 per cent. Caesarean section delivery continues to be more common among privately than publicly insured mothers. The changing pattern in type of delivery is evident in both groups between 2000 and 2004. Among privately insured mothers the rate of normal vaginal birth decreased from 55.0 to 50.3 per cent and the caesarean section rate increased from 28.2 to 35.0 per cent. Among publicly insured mothers the rate of normal vaginal birth decreased from 71.8 to 67.8 per cent and the caesarean section rate increased from 18.5 to 23.4 per cent.

Since 2001, the rate of low birth weight (less than 2,500 grams) has been steady at about 6 per cent. The rate was 6.3 per cent in 2005. The percentage of babies born prematurely (less than 37 weeks gestation) has remained stable at about 7 per cent and was 7.2 per cent in 2005 .

The perinatal mortality rate varied from 8.6 to 9.6 per 1,000 births over the 5 year period. About two-thirds of all perinatal deaths were stillbirths and one-third were neonatal deaths.

In the period 1990-2004, 155 deaths were reported among pregnant women or women who gave birth less than 6 weeks previously. The number of deaths per year has gradually declined with less than 10 deaths per year reported since 2001 .

\section{Aboriginal and Torres Strait Islander Mothers and Babies}

Between 2001 and 2005, the proportion of Aboriginal and Torres Strait Islander mothers who commenced antenatal care at less than 20 weeks gestation increased from 64.7 to 74.9 per cent. This compares with 88.4 per cent of non-Aboriginal or Torres Strait Islander mothers who commenced antenatal care at less than 20 weeks gestation in 2005 .

In 2005, 55.3 per cent of Aboriginal and Torres Strait Islander mothers reported smoking at some time during pregnancy, compared with 59.0 per cent in 2001.

Since 2001, the rates of low birth weight (less than 2,500 grams) and prematurity (less than 37 weeks gestation) in Aboriginal and Torres Strait Islander babies have been over 10 per cent. These rates are one and a half times to 2 times higher than the rates for NSW overall. The perinatal mortality rate among babies born to Aboriginal and Torres Strait Islander mothers was 15.2 per 1,000 in 2005 , higher than the rate of 8.6 per 1,000 experienced by babies born to non-Aboriginal or Torres Strait Islander mothers. 


\section{Neonatal Intensive Care}

There were 2,257 infants registered in the Neonatal Intensive Care Units' Data Collection in 2005 representing a registration rate of 23.8 per 1,000 live births.

Antenatal complications were reported for 87.1 per cent of mothers. The proportion of women receiving antenatal corticosteroids for lung maturation has increased each year since 1992, with 87 per cent of mothers receiving steroids in 2005.

Thirty-six per cent of infants registered in 2005 were born following a booked tertiary centre birth and 33.1 per cent were born following maternal transfer. Twenty-nine per cent were transferred to a tertiary centre following birth and 3.2 per cent were transferred from one tertiary centre to another during the first day of life.

Most infants (79 per cent) were from a singleton pregnancy, 18.3 per cent were from a twin pregnancy, 2.3 per cent were from a triplet pregnancy and 0.3 per cent were from a quadruplet pregnancy.

Seventy-four per cent of infants registered during 2005 were preterm (less than 37 weeks gestation), 39.7 per cent were very preterm (less than 32 weeks gestation) and 10.8 per cent were extremely preterm (less than 28 weeks gestation). Nearly one in six (15.6 per cent) infants had a major or minor congenital anomaly.

Infants with major congenital anomalies were excluded from the analysis of mortality and morbidity. The majority of infants registered in 2005 (86.7 per cent) received assisted ventilation (intermittent mandatory ventilation or continuous positive airways pressure ventilation). The main indication for assisted ventilation varied with gestational age: respiratory distress syndrome, immature lung and transient tachypnoea were more common among preterm groups, whereas meconium aspiration and perinatal asphyxia were more common in term infants.

Proven systemic infection was present in 10.5 per cent of infants. Severe grades (Grade 3 or 4 ) of retinopathy of prematurity were present in 4.3 per cent of infants less than 32 weeks gestation, of whom 74.2 per cent had laser therapy to prevent retinal detachment. Surfactant was given to 36.5 per cent of infants; the majority ( 52.4 per cent) of ventilated infants with a diagnosis of Respiratory Distress Syndrome received surfactant.

Overall, 94 per cent of infants without a major congenital anomaly survived to six-months of age. Survival improved with gestational age up to 36 weeks after which it decreased slightly. Of the infants who died, most (68.4 per cent) died at less than one week of age and a further 21.1 per cent died at less than 29 days of age. The six-month survival rate for infants born at all gestational ages was similar for those born in a tertiary centre and those born in a non-tertiary centre.

\section{Extremely Preterm Follow up}

From 1998 to 2002, 69.3 per cent of 22 to 28 weeks gestation infants were liveborn. Eighty-nine per cent of liveborn infants were admitted to a neonatal intensive care unit and 77.2 per cent of neonatal intensive care unit admissions survived to hospital discharge. A further 1.7 per cent of children died after hospital discharge and before their follow up appointment.

There were 1,490 children available for follow up at 2-3 years of age, corrected for prematurity. The follow up rate was 79.3 per cent. The median (25th, 75 th) age of assessment was $35.6(29.5,36.8)$ months.

Of the 1,182 children with information at 2-3 years of age, corrected for prematurity, 10.8 per cent had cerebral palsy, 0.9 per cent were bilaterally blind, 5.3 per cent were bilaterally deaf and 10.8 per cent had a moderate to severe developmental delay.

Overall 16.8 per cent of children had a moderate to severe functional disability due to cerebral palsy, bilateral blindness, deafness requiring bilateral hearing aids or cochlear implants or developmental delay more than 2 standard deviations below the mean on a standardized psychological assessment.

\section{Birth defects}

About 2,000 infants are born with birth defects each year in NSW. In 1999-2005, defects of the cardiovascular system were most commonly reported, followed by defects of the musculoskeletal system and defects of the genito-urinary system. This is a similar pattern to previous years.

Birth defects were more common among premature infants compared to full term infants, and among male infants compared to female infants. The rate of birth defects increases with increasing maternal age, especially after age 35 . However, as most babies are born to mothers aged less than 35 years, the majority of babies with birth defects were born to younger mothers.

\section{Perinatal deaths}

Confidential reports on 630 perinatal deaths in 2005 were reviewed. Overall, 174 (27.6 per cent) perinatal deaths reviewed for 2005 were unexplained. The next most common obstetric antecedents of death were spontaneous preterm labour ( $n=119,18.9$ per cent), fetal abnormality $(n=114,18.1$ per cent) and antepartum haemorrhage $(n=51$, 8.1 per cent). Post-mortem examinations were carried out in 37.7 per cent of all perinatal deaths.

The most common cause of neonatal death was extreme prematurity ( $n=97,43.1$ per cent), followed by congenital abnormalities ( $n=47,20.9$ per cent). 